\title{
Investigating the association between diet and risk of head and neck cancer in Taiwan
}

\author{
Chan-Chi Chang ${ }^{1,2, *}$, Wei-Ting Lee ${ }^{1,2, *}$, Yao-Chou Lee $^{2,3}$, Cheng-Chih Huang ${ }^{1}$, Chun- \\ Yen Ou ${ }^{1}$, Yu-Hsuan Lin ${ }^{1}$, Jehn-Shyun Huang ${ }^{4}$, Tung-Yiu Wong ${ }^{4}$, Ken-Chung Chen ${ }^{4}$, \\ Jenn-Ren Hsiao ${ }^{1,2}$, Yu-Cheng Lu' ${ }^{1}$, Sen-Tien Tsai ${ }^{1}$, Yu-Hsuan Lai ${ }^{2,5}$, Yuan-Hua Wu ${ }^{5}$, \\ Wei-Ting Hsueh ${ }^{5}$, Chia-Jui Yen ${ }^{6}$, Shang-Yin Wu ${ }^{2,6}$, Jang-Yang Chang ${ }^{6,7}$, Sheen-Yie \\ Fang $^{1}$, Jiunn-Liang $\mathrm{Wu}^{1}{ }^{1}$, Chen-Lin Lin ${ }^{8}$, Ya-Ling Weng ${ }^{7}$, Han-Chien Yang ${ }^{7}$, Yu-Shan \\ Chen $^{1}$ and Jeffrey S. Chang ${ }^{7}$ \\ ${ }^{1}$ Department of Otolaryngology, National Cheng Kung University Hospital, College of Medicine, National Cheng Kung \\ University, Tainan 70456, Taiwan \\ ${ }^{2}$ Institute of Clinical Medicine, College of Medicine, National Cheng Kung University, Tainan 70456, Taiwan \\ ${ }^{3}$ Division of Plastic and Reconstructive Surgery, Department of Surgery, National Cheng Kung University Hospital, College of \\ Medicine, National Cheng Kung University, Tainan 70456, Taiwan \\ ${ }^{4}$ Department of Stomatology, National Cheng Kung University Hospital, College of Medicine, National Cheng Kung University, \\ Tainan 70456, Taiwan \\ ${ }^{5}$ Department of Radiation Oncology, National Cheng Kung University Hospital, College of Medicine, National Cheng Kung \\ University, Tainan 70456, Taiwan \\ ${ }^{6}$ Division of Hematology/Oncology, Department of Internal Medicine, National Cheng Kung University Hospital, College of \\ Medicine, National Cheng Kung University, Tainan 70456, Taiwan \\ ${ }^{7}$ National Institute of Cancer Research, National Health Research Institutes, Tainan 70456, Taiwan \\ ${ }^{8}$ Department of Nursing, National Cheng Kung University Hospital, College of Medicine, National Cheng Kung University, \\ Tainan 70456, Taiwan \\ * These authors have contributed equally to this work \\ Correspondence to: Jeffrey S. Chang, email: jeffreychang@nhri.org.tw
}

Keywords: head and neck cancer, diet, vegetables and fruits, risk factor, Taiwan

Abbreviations: confidence interval (CI); head and neck cancer (HNC); Nutrition and Heath Surveys in Taiwan (NAHSIT); OR (Odds ratio); upper aerodigestive tract (UADT)

Received: July 23, $2017 \quad$ Accepted: September 30, $2017 \quad$ Published: October 24, 2017

Copyright: Chang et al. This is an open-access article distributed under the terms of the Creative Commons Attribution License 3.0 (CC BY 3.0), which permits unrestricted use, distribution, and reproduction in any medium, provided the original author and source are credited.

\section{ABSTRACT}

Most studies reporting an inverse association between the consumption of vegetables and fruits and head and neck cancer (HNC) risk were conducted in Western populations and only a few included East Asians. The current case-control study investigated the association between diet and HNC risk using data of 838 HNC cases and 998 controls from a case-control study of HNC conducted in Taiwan. Each participant was asked about their consumption of fresh vegetables, pickled vegetables, fresh fruits, citrus fruits, meat, processed meat, fish, egg, and dairy products. Unconditional logistic regression was performed to estimate the odds ratio (OR) and $95 \%$ confidence interval (CI) of HNC risk associated with each food category, adjusted for sex, age, education, and use of alcohol, betel quid and cigarette. An inverse association was observed between HNC risk and daily intake of fresh vegetables (OR $=0.44,95 \%$ CI: $0.20-0.95, p$-trend $=0.002)$ or fruits $(O R=0.55$, 95\% CI: $0.43-0.72, p$-trend $=0.00001)$. Individuals who did not consume fresh fruits and vegetables daily had more than double the risk of HNC compared to those with daily intake of vegetables and fruits $(O R=2.24,95 \% \mathrm{CI}: 1.54-3.25)$. The results of 


\begin{abstract}
the current study supported an inverse association between the consumption of fresh vegetables and fruits and HNC risk. In addition to cessation of cigarette smoking and betel quid chewing and reduction of alcohol drinking, a public health campaign for preventing the occurrence of HNC should promote a healthy diet that contains plenty of fresh vegetables and fruits.
\end{abstract}

\section{INTRODUCTION}

Head and neck cancer (HNC) consists of cancers of the oral cavity, oropharynx, hypopharynx, and larynx and together these cancers make up the fifth leading cancer in the world, with approximately 600,000 incident cases diagnosed worldwide annually [1]. HNC is also one of the leading cancers in Taiwan, particularly among men. In 2014, oral, oropharyngeal, and hypopharyngeal cancers together ranked as the sixth most common cancer in Taiwan and as the fourth most common cancer among Taiwanese men [2]. The risk factors of HNC are well-established and the occurrence of most HNCs can be attributed to the consumption of alcohol, betel quid, and cigarette [3]. In addition, the incidence of human papillomavirus-associated oropharyngeal cancer has been increasing [4]. In contrast to the well-known risk factors of $\mathrm{HNC}$, less is known about the factors associated with a reduced $\mathrm{HNC}$ risk. The only factor that has been consistently associated with a reduced $\mathrm{HNC}$ risk is the consumption of fruits and vegetables [5]. In a pooled analysis of 14,520 HNC cases and 22,737 controls from studies conducted in North American, South America, Europe, and Asia, the highest quartile of fruit (odds ratio $(\mathrm{OR})=0.52,95 \%$ confidence interval $(\mathrm{CI}): 0.43-0.62, p$ trend $<0.01)$ and vegetable $(\mathrm{OR}=0.66,95 \% \mathrm{CI}: 0.49$ $0.90, p$-trend $=0.01)$ consumption was associated with a lower HNC risk compared to the lowest quartile [6].

Most studies published to date on the role of diet in $\mathrm{HNC}$ risk focused on Western populations and only a few originated from East Asian countries. Compared with a Western diet, the Asian diet tends to contain more vegetables and fruits and less meat. Although Taiwan has experienced westernization of lifestyle in recent decades, according to the 1993-1996 and 2005-2008 Nutrition and Heath Surveys in Taiwan (NAHSIT), Taiwanese people increased their consumption of vegetables and fruits, soy products, fish, whole grain, nuts and seeds, while decreasing the consumption of red meat, carbohydrates, and sodium-containing foods, and the intake of dairy products has remained low [7]. Given the difference in dietary patterns between Taiwanese and Western populations, it is interesting to consider whether diet plays a similar role in $\mathrm{HNC}$ occurring in Taiwan compared to that in Western populations. Furthermore, in addition to alcohol and cigarette, which are the major contributors to $\mathrm{HNC}$ in Western countries, betel quid is another major cause of HNC in Taiwan [8]. It is unclear whether the relationship between diet and $\mathrm{HNC}$, particularly the inverse association between vegetables and fruits and
HNC, might differ according to the status of betel quid use.

The aim of the current study was to investigate the association between diet and $\mathrm{HNC}$ using data from an ongoing hospital-based case-control study of HNC in Taiwan. In addition, we examined whether the relationship between diet and $\mathrm{HNC}$ risk might differ according to the use of alcohol, betel quid, and cigarette.

\section{RESULTS}

$\mathrm{HNC}$ cases and controls were not significantly different $(p>0.05)$ in mean age and distribution of sex (Table 1). Controls had significantly more years of education compared to the cases $(p<0.0001)$, with $65 \%$ of the controls having completed at least a high school education vs. $43 \%$ of the cases. More HNC cases were users of alcohol, betel quid, or cigarette compared to the controls $(p<0.0001)$.

Table 2 presents the association between plantbased food and HNC risk. Consumption of fresh vegetables or fresh fruits was associated with a significantly reduced $\mathrm{HNC}$ risk with a significant $p$-trend $=0.002$ for fresh vegetables and a $p$-trend $=0.00001$ for fresh fruits. Consumption of pickled vegetables or citrus fruits was not associated with HNC risk. In the subsite analysis, consumption of fresh vegetables was associated with a reduced risk of oral cancer and pharyngeal cancer but not laryngeal cancer. Consumption of fruits was associated with a reduced risk across all subsites of HNC.

Table 3 presents the association between animalbased food and HNC risk. Overall, no significant association was observed between HNC risk and meat, processed meat, fish, egg, or dairy products. For the subsite analyses, the only significant finding was the significant inverse association between consumption of egg and pharyngeal cancer.

Compared to individuals with daily intake of fresh vegetables and fruits, those who only had daily consumption of fresh vegetables had a 1.45 times increase in $\mathrm{HNC}$ risk (OR $=1.45,95 \% \mathrm{CI}: 1.14-1.84)$, whereas those who only had daily intake of fresh fruits did not have a significant increase in $\mathrm{HNC}$ risk $(\mathrm{OR}=1.12,95 \% \mathrm{CI}$ : 0.44-2.84) (Table 4). The highest HNC risk was observed for those without daily intake of both fresh vegetables and fruits $(\mathrm{OR}=2.24 .95 \% \mathrm{CI}$ : $1.54-3.25)$.

The association between consumption of fresh vegetables or fruits and $\mathrm{HNC}$ risk did not vary significantly ( $p$-heterogeneity $>0.05$ ) by the use of alcohol, betel quid, 
Table 1: Demographic and lifestyle characteristics of the head and neck cancer patients and control subjects

\begin{tabular}{|c|c|c|c|}
\hline Characteristics & $\begin{array}{c}\text { Case } \\
\mathrm{N}=\mathbf{8 3 8} \\
\mathrm{n}(\%)\end{array}$ & $\begin{array}{c}\text { Control } \\
\mathrm{N}=998 \\
\mathrm{n}(\%)\end{array}$ & $\boldsymbol{P}$ \\
\hline \multicolumn{4}{|l|}{ Age (years) } \\
\hline Mean (SE) & $55.5(0.4)$ & $54.7(0.3)$ & 0.09 \\
\hline \multicolumn{4}{|l|}{ Sex } \\
\hline Men & $786(93.8)$ & $948(95.0)$ & 0.27 \\
\hline Women & $52(6.2)$ & $50(5.0)$ & \\
\hline \multicolumn{4}{|l|}{ Education } \\
\hline$\leq$ Elementary school & $228(27.2)$ & $170(17.0)$ & $<0.0001$ \\
\hline Junior high & $250(29.8)$ & $177(17.7)$ & \\
\hline High school/Technical school & $276(33.0)$ & $359(36.0)$ & \\
\hline Some college or more & $84(10.0)$ & $292(29.3)$ & \\
\hline \multicolumn{4}{|l|}{ Alcohol drinking } \\
\hline Never + occasional & $273(32.6)$ & $543(54.4)$ & $<0.0001$ \\
\hline Former regular & $116(13.8)$ & $113(11.3)$ & \\
\hline Current regular & $449(53.6)$ & $342(34.3)$ & \\
\hline Never & $245(29.3)$ & $476(47.7)$ & $<0.0001$ \\
\hline 1 drink or less per month & $28(3.3)$ & $66(6.6)$ & \\
\hline 1-2 drinks per week & $37(4.4)$ & $66(6.6)$ & \\
\hline 3-5 drinks per week & $48(5.7)$ & $71(7.1)$ & \\
\hline Daily drinkers & $461(55.0)$ & $312(31.3)$ & \\
\hline Unknown & $19(2.3)$ & $7(0.7)$ & \\
\hline \multicolumn{4}{|l|}{ Betel quid chewing } \\
\hline Never & $241(28.8)$ & $713(71.5)$ & $<0.0001$ \\
\hline Former & $317(37.8)$ & $185(18.5)$ & \\
\hline Current & $280(33.4)$ & $99(9.9)$ & \\
\hline Unknown & $0(0.0)$ & $1(0.1)$ & \\
\hline Never & $241(28.8)$ & $713(71.5)$ & $<0.0001$ \\
\hline 0.1-9.9 pack-years & $122(14.6)$ & $100(10.0)$ & \\
\hline 10.0-19.9 pack-years & $105(12.5)$ & $51(5.1)$ & \\
\hline 20.0-29.9 pack-years & $93(11.1)$ & $40(4.0)$ & \\
\hline 30.0 or more pack-years & $256(30.5)$ & $90(9.0)$ & \\
\hline Unknown & $21(2.5)$ & $4(0.4)$ & \\
\hline Pack-years (SE) & $26.6(1.3)$ & $7.6(0.7)$ & $<0.0001$ \\
\hline
\end{tabular}




\begin{tabular}{lccc}
\hline Characteristics & $\begin{array}{c}\text { Case } \\
\mathbf{N}=\mathbf{8 3 8} \\
\mathbf{n}(\mathbf{\%})\end{array}$ & $\begin{array}{c}\text { Control } \\
\mathbf{N = 9 9 8} \\
\mathbf{n}(\mathbf{\%})\end{array}$ & $\boldsymbol{P}$ \\
\hline Cigarette smoking & & & $<0.0001$ \\
Never & $114(13.6)$ & $326(32.7)$ & \\
Former & $158(18.9)$ & $205(20.5)$ & \\
Current & $565(67.4)$ & $466(46.7)$ & $<0.0001$ \\
Unknown & $1(0.1)$ & $1(0.1)$ & \\
& & $326(32.7)$ & \\
Never & $114(13.6)$ & $78(7.8)$ & \\
$0.1-9.9$ pack-years & $39(4.6)$ & $128(13.3)$ & \\
$10.0-19.9$ pack-years & $81(9.7)$ & $327(32.8)$ & $<0.0001$ \\
$20.0-29.9$ pack-years & $140(16.7)$ & $6(0.6)$ & \\
30.0 or more pack-years & $453(54.1)$ & $23.0(0.8)$ & \\
Unknown & $11(1.3)$ & & \\
Pack-years (SE) & $35.8(1.0)$ & & \\
\hline
\end{tabular}

Abbreviations: N: number, SE: standard error.

Table 2: The association between plant-based food and head and neck cancer

\begin{tabular}{|c|c|c|c|c|c|c|c|c|c|}
\hline Diet & $\begin{array}{c}\text { Control } \\
\mathbf{N}=998 \\
\text { n }(\%)\end{array}$ & $\begin{array}{c}\text { Head and } \\
\text { neck cancer } \\
\mathbf{N}=838 \\
\text { n (\%) }\end{array}$ & $\begin{array}{c}\text { OR } \\
(95 \% \mathrm{CI})^{\mathrm{a}}\end{array}$ & $\begin{array}{c}\text { Oral cancer } \\
\mathbf{N}=\mathbf{5 2 5} \\
\mathbf{n}(\%)\end{array}$ & $\begin{array}{c}\text { OR } \\
(95 \% \mathrm{CI})^{\mathrm{a}}\end{array}$ & $\begin{array}{c}\text { Pharyngeal } \\
\text { cancer } \\
\mathbf{N}=214 \\
\text { n (\%) }\end{array}$ & $\begin{array}{c}\text { OR } \\
(95 \% \mathrm{CI})^{\mathrm{a}}\end{array}$ & $\begin{array}{c}\text { Laryngeal } \\
\text { cancer } \\
\mathbf{N}=99 \\
\mathbf{n}(\%)\end{array}$ & $\begin{array}{c}\text { OR } \\
(95 \% \mathrm{CI})^{\mathrm{a}}\end{array}$ \\
\hline \multicolumn{10}{|l|}{ Fresh vegetables } \\
\hline$\leqq$ once/week & $11(1.1)$ & $31(3.7)$ & Reference & $20(3.8)$ & Reference & $9(4.2)$ & Reference & $2(2.1)$ & Reference \\
\hline 2-4 times/week & $74(7.4)$ & $124(14.8)$ & $\begin{array}{c}0.66 \\
(0.29-1.53)\end{array}$ & $\begin{array}{c}77 \\
(14.7)\end{array}$ & $\begin{array}{c}0.59 \\
(0.24-1.45)\end{array}$ & $31(14.6)$ & $\begin{array}{c}0.53 \\
(0.16-1.77)\end{array}$ & $16(16.3)$ & $\begin{array}{c}1.47 \\
(0.26-8.41)\end{array}$ \\
\hline \multirow[t]{2}{*}{ Daily } & $913(91.5)$ & $681(81.5)$ & $\begin{array}{c}0.44 \\
(0.20-0.95)\end{array}$ & $428(81.5)$ & $\begin{array}{c}0.38 \\
(0.17-0.87)\end{array}$ & $173(81.2)$ & $\begin{array}{c}0.37 \\
(0.12-1.12)\end{array}$ & $80(81.6)$ & $\begin{array}{c}0.87 \\
(0.17-4.61)\end{array}$ \\
\hline & & & $\begin{array}{c}\text { P-trend }= \\
0.002\end{array}$ & & $\begin{array}{c}\text { P-trend }= \\
0.002\end{array}$ & & $\begin{array}{c}\text { P-trend }= \\
0.03\end{array}$ & & $\begin{array}{c}\text { P-trend }= \\
0.21\end{array}$ \\
\hline \multicolumn{10}{|l|}{$\begin{array}{l}\text { Pickled } \\
\text { vegetables }\end{array}$} \\
\hline$\leqq$ once/week & $935(94.0)$ & 789 (94.5) & Reference & $500(95.6)$ & Reference & 198 (92.9) & Reference & 91 (91.9) & Reference \\
\hline 2-4 times/week & $49(4.9)$ & $40(4.8)$ & $\begin{array}{c}0.87 \\
(0.53-1.42)\end{array}$ & $21(4.0)$ & $\begin{array}{c}0.79 \\
(0.44-1.43)\end{array}$ & $14(6.6)$ & $\begin{array}{c}1.31 \\
(0.65-2.67)\end{array}$ & $5(5.1)$ & $\begin{array}{c}0.93 \\
(0.32-2.66)\end{array}$ \\
\hline \multirow[t]{2}{*}{ Daily } & $11(1.1)$ & $6(0.7)$ & $\begin{array}{c}0.57 \\
(0.19-1.77)\end{array}$ & $1(0.4)$ & $\begin{array}{c}0.28 \\
(0.05-1.45)\end{array}$ & $1(0.5)$ & $\begin{array}{c}0.28 \\
(0.03-2.49)\end{array}$ & $3(3.0)$ & $\begin{array}{c}2.00 \\
(0.49-8.20)\end{array}$ \\
\hline & & & $\begin{array}{c}\text { P-trend }= \\
0.29\end{array}$ & & $\begin{array}{c}\text { P-trend }= \\
0.11\end{array}$ & & $\begin{array}{c}\text { P-trend }= \\
0.81\end{array}$ & & $\begin{array}{c}\text { P-trend }= \\
0.51\end{array}$ \\
\hline \multicolumn{10}{|l|}{ Fresh fruits } \\
\hline$\leqq$ once/week & $221(22.2)$ & 348 (41.7) & Reference & $213(40.7)$ & Reference & $92(43.2)$ & Reference & 43 (43.9) & Reference \\
\hline 2-4 times/week & $263(26.4)$ & $236(28.3)$ & $\begin{array}{c}0.75 \\
(0.57-0.98)\end{array}$ & $152(29.0)$ & $\begin{array}{c}0.79 \\
(0.57-1.08)\end{array}$ & $58(27.2)$ & $\begin{array}{c}0.71 \\
(0.46-1.09)\end{array}$ & $26(26.5)$ & $\begin{array}{c}0.60 \\
(0.34-1.06)\end{array}$ \\
\hline
\end{tabular}

(Continued) 


\begin{tabular}{|c|c|c|c|c|c|c|c|c|c|}
\hline Diet & $\begin{array}{c}\text { Control } \\
\mathbf{N}=998 \\
\text { n }(\%)\end{array}$ & $\begin{array}{c}\text { Head and } \\
\text { neck cancer } \\
\mathbf{N}=\mathbf{8 3 8} \\
\mathbf{n}(\%)\end{array}$ & $\begin{array}{c}\text { OR } \\
(95 \% \mathrm{CI})^{\mathrm{a}}\end{array}$ & $\begin{array}{c}\text { Oral cancer } \\
\mathbf{N}=\mathbf{5 2 5} \\
\mathbf{n}(\%)\end{array}$ & $\begin{array}{c}\text { OR } \\
(95 \% \mathrm{CI})^{\mathrm{a}}\end{array}$ & $\begin{array}{c}\text { Pharyngeal } \\
\text { cancer } \\
\mathrm{N}=\mathbf{2 1 4} \\
\mathrm{n}(\%)\end{array}$ & $\begin{array}{c}\text { OR } \\
(95 \% \mathrm{CI})^{\mathrm{a}}\end{array}$ & $\begin{array}{c}\text { Laryngeal } \\
\text { cancer } \\
\mathbf{N}=99 \\
\mathbf{n}(\%)\end{array}$ & $\begin{array}{c}\text { OR } \\
(95 \% \text { CI })^{\mathrm{a}}\end{array}$ \\
\hline \multirow[t]{2}{*}{ Daily } & $513(51.4)$ & $251(30.0)$ & $\begin{array}{c}0.55 \\
(0.43-0.72)\end{array}$ & $159(30.3)$ & $\begin{array}{c}0.59 \\
(0.43-0.80)\end{array}$ & $63(29.6)$ & $\begin{array}{c}0.58 \\
(0.38-0.89)\end{array}$ & $29(29.6)$ & $\begin{array}{c}0.39 \\
(0.22-0.69)\end{array}$ \\
\hline & & & $\begin{array}{c}\text { P-trend }= \\
0.00001\end{array}$ & & $\begin{array}{c}\text { P-trend }= \\
0.0006\end{array}$ & & $\begin{array}{c}\text { P-trend }= \\
0.01\end{array}$ & & $\begin{array}{c}\text { P-trend }= \\
0.001\end{array}$ \\
\hline
\end{tabular}

\section{Citrus fruits}

\begin{tabular}{|c|c|c|c|c|c|c|c|c|c|}
\hline$\leqq$ once/week & $893(89.5)$ & 778 (93.2) & Reference & $482(92.0)$ & Reference & $203(95.3)$ & Reference & 93 (94.9) & Reference \\
\hline 2-4 times/week & $68(6.8)$ & $43(5.1)$ & $\begin{array}{c}1.02 \\
(0.65-1.60)\end{array}$ & $31(5.9)$ & $\begin{array}{c}1.17 \\
(0.70-1.95)\end{array}$ & $8(3.8)$ & $\begin{array}{c}0.68 \\
(0.30-1.55)\end{array}$ & $4(4.1)$ & $\begin{array}{c}0.78 \\
(0.26-2.31)\end{array}$ \\
\hline \multirow[t]{2}{*}{ Daily } & $37(3.7)$ & $14(1.7)$ & $\begin{array}{c}0.53 \\
(0.26-1.07)\end{array}$ & $11(2.1)$ & $\begin{array}{c}0.70 \\
(0.32-1.54)\end{array}$ & $2(0.9)$ & $\begin{array}{c}0.37 \\
(0.08-1.60)\end{array}$ & $1(1.0)$ & $\begin{array}{c}0.25 \\
(0.03-2.00)\end{array}$ \\
\hline & & & $\begin{array}{c}\text { P-trend }= \\
0.16\end{array}$ & & $\begin{array}{c}\text { P-trend }= \\
0.71\end{array}$ & & $\begin{array}{c}\text { P-trend }= \\
0.11\end{array}$ & & $\begin{array}{c}\text { P-trend }= \\
0.17\end{array}$ \\
\hline
\end{tabular}

Abbreviations: CI: confidence interval, N: number, OR: odds ratio.

${ }^{a}$ OR and 95\% CI were calculated using unconditional logistic regression, adjusted for sex, age, education, cigarette smoking (pack-year categories), betel quid chewing (pack-year categories), and alcohol drinking (frequency).

Table 3: The association between animal-based food and head and neck cancer

\begin{tabular}{|c|c|c|c|c|c|c|c|c|c|}
\hline Diet & $\begin{array}{l}\text { Control } \\
\mathrm{N}=998 \\
\mathrm{n}(\%)\end{array}$ & $\begin{array}{c}\text { Head and } \\
\text { neck cancer } \\
\mathbf{N}=\mathbf{8 3 8} \\
\text { n (\%) }\end{array}$ & $\begin{array}{c}\text { OR } \\
(95 \% \mathrm{CI})^{\mathrm{a}}\end{array}$ & $\begin{array}{c}\text { Oral cancer } \\
\mathbf{N}=\mathbf{5 2 5} \\
\mathbf{n}(\%)\end{array}$ & $\begin{array}{c}\text { OR } \\
(95 \% \mathrm{CI})^{\mathrm{a}}\end{array}$ & $\begin{array}{c}\text { Pharyngeal } \\
\text { cancer } \\
\mathbf{N}=\mathbf{2 1 4} \\
\mathbf{n}(\%)\end{array}$ & $\begin{array}{c}\text { OR } \\
\mid(95 \% \mathrm{CI})^{\mathrm{a}}\end{array}$ & $\begin{array}{c}\text { Laryngeal } \\
\text { cancer } \\
\mathbf{N}=99 \\
\mathbf{n}(\%)\end{array}$ & $\begin{array}{c}\text { OR } \\
(95 \% \text { CI })^{a}\end{array}$ \\
\hline \multicolumn{10}{|l|}{ Meat } \\
\hline @once/week & $94(9.4)$ & $74(8.8)$ & Reference & $46(8.8)$ & Reference & $18(8.4)$ & Reference & $10(10.1)$ & Reference \\
\hline 2-4 times/week & $232(23.3)$ & $202(24.2)$ & $\begin{array}{c}0.99 \\
(0.65-1.50)\end{array}$ & $123(23.5)$ & $\begin{array}{c}0.90 \\
(0.55-1.45)\end{array}$ & $53(24.8)$ & $\begin{array}{c}1.29 \\
(0.65-2.54)\end{array}$ & $26(26.3)$ & $\begin{array}{c}0.89 \\
(0.38-2.05)\end{array}$ \\
\hline \multirow[t]{2}{*}{ Daily } & $672(67.3)$ & $560(67.0)$ & $\begin{array}{c}1.02 \\
(0.70-1.51)\end{array}$ & 354 (67.7) & $\begin{array}{c}0.89 \\
(0.57-1.39)\end{array}$ & $143(66.8)$ & $\begin{array}{c}1.25 \\
(0.66-2.37)\end{array}$ & $63(63.6)$ & $\begin{array}{c}0.96 \\
(0.44-2.09)\end{array}$ \\
\hline & & & $\begin{array}{c}\text { P-trend }= \\
0.83\end{array}$ & & $\begin{array}{c}\text { P-trend }= \\
0.67\end{array}$ & & $\begin{array}{c}\text { P-trend }= \\
0.63\end{array}$ & & $\begin{array}{c}\text { P-trend }= \\
0.96\end{array}$ \\
\hline \multicolumn{10}{|l|}{ Processed meat } \\
\hline$\leqq$ once/week & $873(87.5)$ & $724(86.8)$ & Reference & $446(85.3)$ & Reference & $186(86.9)$ & Reference & $92(94.8)$ & Reference \\
\hline 2-4 times/week & $102(10.2)$ & $92(11.0)$ & $\begin{array}{c}1.02 \\
(0.72-1.44)\end{array}$ & $65(12.4)$ & $\begin{array}{c}1.17 \\
(0.80-1.73)\end{array}$ & $24(11.2)$ & $\begin{array}{c}0.92 \\
(0.53-1.59)\end{array}$ & $3(3.1)$ & $\begin{array}{c}0.33 \\
(0.10-1.09)\end{array}$ \\
\hline \multirow[t]{2}{*}{ Daily } & $23(2.3)$ & $18(2.2)$ & $\begin{array}{c}0.90 \\
(0.44-1.84)\end{array}$ & $12(2.3)$ & $\begin{array}{c}0.96 \\
(0.42-2.18)\end{array}$ & $4(1.9)$ & $\begin{array}{c}0.82 \\
(0.25-2.73)\end{array}$ & $2(2.1)$ & $\begin{array}{c}0.51 \\
(0.09-2.77)\end{array}$ \\
\hline & & & $\begin{array}{c}\text { P-trend }= \\
0.91\end{array}$ & & $\begin{array}{c}\text { P-trend }= \\
0.61\end{array}$ & & $\begin{array}{c}\text { P-trend }= \\
0.67\end{array}$ & & $\begin{array}{c}\text { P-trend }= \\
0.08\end{array}$ \\
\hline
\end{tabular}

Fish

\begin{tabular}{|c|c|c|c|c|c|c|c|c|c|}
\hline$\leqq$ once/week & $179(17.9)$ & $153(18.3)$ & Reference & $91(17.4)$ & Reference & $48(22.4)$ & Reference & $14(14.4)$ & Reference \\
\hline 2-4 times/week & $353(35.4)$ & $243(29.1)$ & $\begin{array}{c}0.75 \\
(0.55-1.03)\end{array}$ & $156(29.8)$ & $\begin{array}{c}0.81 \\
(0.56-1.17)\end{array}$ & $63(29.5)$ & $\begin{array}{c}0.65 \\
(0.40-1.06)\end{array}$ & $24(24.8)$ & $\begin{array}{c}0.70 \\
(0.34-1.46)\end{array}$ \\
\hline \multirow[t]{2}{*}{ Daily } & $466(46.7)$ & $439(52.6)$ & $\begin{array}{c}0.94 \\
(0.70-1.27)\end{array}$ & $277(52.8)$ & $\begin{array}{c}1.03 \\
(0.73-1.45)\end{array}$ & $103(48.1)$ & $\begin{array}{c}0.72 \\
(0.46-1.15)\end{array}$ & $59(60.8)$ & $\begin{array}{c}0.83 \\
(0.43-1.61)\end{array}$ \\
\hline & & & $\begin{array}{c}\text { P-trend }= \\
0.84\end{array}$ & & $\begin{array}{c}\text { P-trend }= \\
0.51\end{array}$ & & $\begin{array}{c}\text { P-trend }= \\
0.29\end{array}$ & & $\begin{array}{c}\text { P-trend }= \\
0.82\end{array}$ \\
\hline
\end{tabular}

(Continued) 


\begin{tabular}{|c|c|c|c|c|c|c|c|c|c|}
\hline Diet & $\begin{array}{c}\text { Control } \\
\mathrm{N}=998 \\
\text { n }(\%)\end{array}$ & $\begin{array}{c}\text { Head and } \\
\text { neck cancer } \\
\mathbf{N}=\mathbf{8 3 8} \\
\mathbf{n}(\%)\end{array}$ & $\begin{array}{c}\text { OR } \\
(95 \% \text { CI })^{a}\end{array}$ & $\begin{array}{c}\text { Oral cancer } \\
\text { N = 525 } \\
\text { n }(\%)\end{array}$ & $\begin{array}{c}\text { OR } \\
(95 \% \text { CI })^{a}\end{array}$ & $\begin{array}{c}\text { Pharyngeal } \\
\text { cancer } \\
\text { N=214 } \\
\text { n (\%) }\end{array}$ & $\begin{array}{c}\text { OR } \\
\mid(95 \% \mathrm{CI})^{\mathrm{a}}\end{array}$ & $\begin{array}{c}\text { Laryngeal } \\
\text { cancer } \\
\mathbf{N}=99 \\
\mathbf{n}(\%)\end{array}$ & $\begin{array}{c}\text { OR } \\
(95 \% \mathrm{CI})^{\mathrm{a}}\end{array}$ \\
\hline \multicolumn{10}{|l|}{ Egg } \\
\hline$\leqq$ once/week & $254(25.4)$ & $230(27.7)$ & Reference & $124(24.0)$ & Reference & $67(31.3)$ & Reference & $39(39.8)$ & Reference \\
\hline 2-4 times/week & $452(45.3)$ & $386(46.6)$ & $\begin{array}{c}1.02 \\
(0.78-1.33)\end{array}$ & $248(48.0)$ & $\begin{array}{c}1.13 \\
(0.83-1.55)\end{array}$ & $97(45.3)$ & $\begin{array}{c}0.76 \\
(0.50-1.15)\end{array}$ & $41(41.8)$ & $\begin{array}{c}0.84 \\
(0.49-1.43)\end{array}$ \\
\hline \multirow[t]{2}{*}{ Daily } & $292(29.3)$ & $213(25.7)$ & $\begin{array}{c}0.94 \\
(0.70-1.27)\end{array}$ & $145(28.0)$ & $\begin{array}{c}1.16 \\
(0.82-1.65)\end{array}$ & $50(23.4)$ & $\begin{array}{c}0.60 \\
(0.37-0.97)\end{array}$ & $18(18.4)$ & $\begin{array}{c}0.54 \\
(0.28-1.04)\end{array}$ \\
\hline & & & $\begin{array}{c}\text { P-trend }= \\
0.68\end{array}$ & & $\begin{array}{c}\text { P-trend }= \\
0.41\end{array}$ & & $\begin{array}{c}\text { P-trend }= \\
0.04\end{array}$ & & $\begin{array}{c}\text { P-trend }= \\
0.07\end{array}$ \\
\hline \multicolumn{10}{|l|}{ Dairy products } \\
\hline$\leqq$ once/week & $684(68.6)$ & $614(73.3)$ & Reference & $376(71.6)$ & Reference & $164(76.6)$ & Reference & $74(74.7)$ & Reference \\
\hline 2-4 times/week & $144(14.4)$ & $107(12.8)$ & $\begin{array}{c}1.11 \\
(0.81-1.52)\end{array}$ & $70(13.3)$ & $\begin{array}{c}1.19 \\
(0.83-1.71)\end{array}$ & 25 (11.7) & $\begin{array}{c}1.07 \\
(0.63-1.81)\end{array}$ & $12(12.1)$ & $\begin{array}{c}1.18 \\
(0.59-2.37)\end{array}$ \\
\hline \multirow[t]{2}{*}{ Daily } & $170(17.0)$ & $117(14.0)$ & $\begin{array}{c}0.91 \\
(0.67-1.23)\end{array}$ & $79(15.1)$ & $\begin{array}{c}1.02 \\
(0.72-1.45)\end{array}$ & 25 (11.7) & $\begin{array}{c}0.74 \\
(0.44-1.25)\end{array}$ & $13(13.1)$ & $\begin{array}{c}0.76 \\
(0.39-1.49)\end{array}$ \\
\hline & & & $\begin{array}{c}\text { P-trend }= \\
0.71\end{array}$ & & $\begin{array}{c}\text { P-trend }= \\
0.71\end{array}$ & & $\begin{array}{c}\text { P-trend }= \\
0.34\end{array}$ & & $\begin{array}{c}\text { P-trend }= \\
0.54\end{array}$ \\
\hline
\end{tabular}

Abbreviations: CI: confidence interval, N: number, OR: odds ratio.

${ }^{a} \mathrm{OR}$ and $95 \% \mathrm{CI}$ were calculated using unconditional logistic regression, adjusted for sex, age, education, cigarette smoking (pack-year categories), betel quid chewing (pack-year categories), and alcohol drinking (frequency).

Table 4: The association between combination of fresh vegetable and fruit consumption and head and neck cancer

\begin{tabular}{lcccc}
\hline $\begin{array}{l}\text { Daily intake of fresh } \\
\text { vegetables }\end{array}$ & $\begin{array}{c}\text { Daily intake of } \\
\text { fresh fruits }\end{array}$ & $\begin{array}{c}\text { Case } \\
\mathbf{N =} \\
\mathbf{n}(\mathbf{8 3 8}\end{array}$ & $\begin{array}{c}\text { Control } \\
\mathbf{N = 9 9 8} \\
\mathbf{n ~ ( \% )}\end{array}$ & OR (95\% CI) \\
\hline Yes & Yes & $239(28.7)$ & $499(50.1)$ & Reference \\
Yes & No & $440(52.8)$ & $414(41.5)$ & $1.45(1.14-1.84)$ \\
No & Yes & $12(1.4)$ & $14(1.4)$ & $1.12(0.44-2.84)$ \\
No & No & $143(17.1)$ & $70(7.0)$ & $2.24(1.54-3.25)$ \\
\hline
\end{tabular}

${ }^{a}$ OR and 95\% CI were calculated using unconditional logistic regression, adjusted for sex, age, education, cigarette smoking (pack-year categories), betel quid chewing (pack-year categories), and alcohol drinking (frequency).

or cigarette (Table 5). Consumption of fresh vegetables or fruits was inversely associated with $\mathrm{HNC}$ risk regardless of the use of alcohol, betel quid, and cigarette.

\section{DISCUSSION}

In the current analysis, we observed a significant inverse association between $\mathrm{HNC}$ risk and consumption of fresh vegetables or fresh fruits. The inverse association was observed across all subsites of $\mathrm{HNC}$ except for the null association between fresh vegetables and laryngeal cancer. The highest risk of HNC was observed among individuals who lacked daily intake of both vegetables and fruits. The inverse association between consumption of fresh vegetables and fruits did not differ according to the use of alcohol, betel quid, or cigarette.

Consistent with results from published studies [6], the current study showed that consumption of fresh vegetables and fruits was also associated with a reduced HNC risk among Taiwanese. In a pooled analysis $(14,520$ HNC cases and 22,737 controls) by Chuang et al. with populations mostly from Western countries, consumption of vegetables $(\mathrm{OR}=0.66$ for highest vs. lowest quartile, $95 \%$ CI: $0.49-0.90, p$-trend $=0.01)$ and fruits $(\mathrm{OR}=0.52$ for highest vs. lowest quartile, $95 \%$ CI: $0.43-0.62, p$-trend $<0.01$ ) was associated with a reduced HNC risk [6]. Bravi et al. reviewed 24 case-controls studies (20 studies from North and South America, Europe and Australia, 3 
Table 5: The association between fresh vegetables and fruits and head and neck cancer by the use of cigarette, alcohol, and betel quid

\begin{tabular}{|c|c|c|c|c|c|c|}
\hline Diet & $\begin{array}{c}\text { Case } \\
\text { n }(\%)\end{array}$ & $\begin{array}{c}\text { Control } \\
\text { n (\%) }\end{array}$ & OR $(95 \% \text { CI })^{a}$ & $\begin{array}{c}\text { Case } \\
\text { n }(\%)\end{array}$ & $\begin{array}{c}\text { Control } \\
\text { n }(\%)\end{array}$ & OR $(95 \% \text { CI })^{a}$ \\
\hline & \multicolumn{3}{|c|}{ Never cigarette smoker } & \multicolumn{3}{|c|}{ Ever cigarette smoker } \\
\hline \multicolumn{7}{|l|}{ Fresh vegetables } \\
\hline$\leqq$ once per week & $3(2.6)$ & $3(0.9)$ & Reference & $28(3.9)$ & $8(1.2)$ & Reference \\
\hline 2-4 time per week & $14(12.3)$ & $17(5.2)$ & $1.27(0.17-9.66)$ & $109(15.1)$ & $57(8.5)$ & $0.58(0.23-1.46)$ \\
\hline \multirow[t]{3}{*}{ Daily } & $97(85.1)$ & $306(93.9)$ & $0.43(0.07-2.76)$ & $584(81.0)$ & $606(90.3)$ & $0.44(0.19-1.05)$ \\
\hline & \multicolumn{5}{|c|}{$\mathrm{P}$-trend $=0.02$} & $\mathrm{P}$-trend $=0.02$ \\
\hline & \multicolumn{6}{|c|}{ P-heterogeneity $=0.25$} \\
\hline Non-daily & $17(14.9)$ & $20(6.1)$ & Reference & $137(19.0)$ & $65(9.7)$ & Reference \\
\hline \multirow[t]{2}{*}{ Daily } & $97(85.1)$ & $306(93.9)$ & $0.35(0.16-0.77)$ & $584(81.0)$ & $606(90.3)$ & $0.70(0.50-0.99)$ \\
\hline & \multicolumn{6}{|c|}{ P-heterogeneity $=0.13$} \\
\hline \multicolumn{7}{|l|}{ Fresh fruits } \\
\hline$\leqq$ once per week & $27(23.7)$ & $32(9.8)$ & Reference & $320(44.4)$ & $189(28.2)$ & Reference \\
\hline 2-4 time per week & $29(25.4)$ & $78(23.9)$ & $0.48(0.22-1.03)$ & $207(28.8)$ & $185(27.6)$ & $0.80(0.60-1.08)$ \\
\hline \multirow[t]{3}{*}{ Daily } & $58(50.9)$ & $216(66.3)$ & $0.47(0.24-0.94)$ & $193(26.8)$ & $296(44.2)$ & $0.55(0.41-0.74)$ \\
\hline & \multicolumn{5}{|c|}{ P-trend $=0.07$} & P-trend $=0.00007$ \\
\hline & \multicolumn{6}{|c|}{ P-heterogeneity $=0.50$} \\
\hline Non-daily & $56(49.1)$ & $110(33.7)$ & Reference & $527(73.2)$ & $374(55.8)$ & Reference \\
\hline \multirow[t]{3}{*}{ Daily } & $58(50.9)$ & $216(66.3)$ & $0.75(0.46-1.25)$ & $193(26.8)$ & $296(44.2)$ & $0.61(0.47-0.70)$ \\
\hline & \multicolumn{6}{|c|}{ P-heterogeneity $=0.59$} \\
\hline & \multicolumn{4}{|c|}{ Never betel quid chewer } & \multicolumn{2}{|c|}{ Ever betel quid chewer } \\
\hline
\end{tabular}

\section{Fresh vegetables}

\begin{tabular}{|c|c|c|c|c|c|c|}
\hline$\leqq$ once per week & $6(2.5)$ & $5(0.7)$ & Reference & $25(4.2)$ & $6(2.1)$ & Reference \\
\hline 2-4 time per week & $31(12.9)$ & $41(5.7)$ & $0.65(0.16-2.61)$ & $93(15.6)$ & $33(11.6)$ & $0.70(0.26-1.89)$ \\
\hline \multirow[t]{3}{*}{ Daily } & $204(84.6)$ & $667(93.6)$ & $0.30(0.08-1.09)$ & $477(80.2)$ & $245(86.3)$ & $0.53(0.21-1.33)$ \\
\hline & \multicolumn{5}{|c|}{ P-trend $=0.0008$} & P-trend $=0.07$ \\
\hline & \multicolumn{6}{|c|}{ P-heterogeneity $=0.23$} \\
\hline Non-daily & $37(15.4)$ & $46(6.4)$ & Reference & $118(19.8)$ & $39(13.7)$ & Reference \\
\hline \multirow[t]{2}{*}{ Daily } & $204(84.6)$ & $667(93.6)$ & $0.43(0.26-0.70)$ & 477 (80.2) & $245(86.3)$ & $0.71(0.47-1.06)$ \\
\hline & \multicolumn{6}{|c|}{ P-heterogeneity $=0.10$} \\
\hline \multicolumn{7}{|l|}{ resh fruits } \\
\hline$\leqq$ once per week & $66(27.4)$ & $117(16.4)$ & Reference & $282(47.5)$ & $104(36.6)$ & Reference \\
\hline 2-4 time per week & $67(27.8)$ & $182(25.6)$ & $0.76(0.49-1.18)$ & $169(28.4)$ & $80(28.2)$ & $0.78(0.55-1.12)$ \\
\hline \multirow[t]{2}{*}{ Daily } & $108(44.8)$ & $413(58.0)$ & $0.60(0.40-0.90)$ & $143(24.1)$ & $100(35.2)$ & $0.53(0.37-0.75)$ \\
\hline & & & P-trend $=0.01$ & & & P-trend $=0.0005$ \\
\hline
\end{tabular}

(Continued) 


\begin{tabular}{|c|c|c|c|c|c|c|}
\hline Diet & $\begin{array}{c}\text { Case } \\
\text { n (\%) }\end{array}$ & $\begin{array}{c}\text { Control } \\
\text { n (\%) }\end{array}$ & OR $(95 \% C I)^{a}$ & $\begin{array}{c}\text { Case } \\
\text { n (\%) }\end{array}$ & $\begin{array}{c}\text { Control } \\
\text { n (\%) }\end{array}$ & OR $(95 \% \text { CI })^{\mathrm{a}}$ \\
\hline & \multicolumn{3}{|c|}{ Never cigarette smoker } & \multicolumn{3}{|c|}{ Ever cigarette smoker } \\
\hline & \multicolumn{6}{|c|}{ P-heterogeneity $=0.86$} \\
\hline Non-daily & $133(55.2)$ & $299(42.0)$ & Reference & $451(75.9)$ & $184(64.8)$ & Reference \\
\hline \multirow[t]{3}{*}{ Daily } & $108(44.8)$ & $413(58.0)$ & $0.70(0.51-0.97)$ & $143(24.1)$ & $100(35.2)$ & $0.59(0.43-0.81)$ \\
\hline & \multicolumn{6}{|c|}{ P-heterogeneity $=0.47$} \\
\hline & \multicolumn{3}{|c|}{ Non-regular alcohol drinker } & \multicolumn{3}{|c|}{ Regular alcohol drinker } \\
\hline \multicolumn{7}{|l|}{ Fresh vegetables } \\
\hline$\leqq$ once per week & $6(2.2)$ & $6(1.1)$ & Reference & $25(4.4)$ & $5(1.1)$ & Reference \\
\hline 2-4 time per week & $32(11.8)$ & $34(6.3)$ & $1.28(0.31-5.26)$ & $92(16.3)$ & $40(8.8)$ & $0.45(0.15-1.37)$ \\
\hline \multirow[t]{3}{*}{ Daily } & $234(86.0)$ & $503(92.6)$ & $0.72(0.19-2.67)$ & $447(79.3)$ & $410(90.1)$ & $0.32(0.11-0.93)$ \\
\hline & & & P-trend $=0.07$ & & & P-trend $=0.01$ \\
\hline & \multicolumn{6}{|c|}{ P-heterogeneity $=0.55$} \\
\hline Non-daily & $38(14.0)$ & $40(7.4)$ & Reference & $117(20.7)$ & $45(9.9)$ & Reference \\
\hline \multirow[t]{2}{*}{ Daily } & $234(86.0)$ & $503(92.6)$ & $0.58(0.34-0.98)$ & $447(79.3)$ & $410(90.1)$ & $0.64(0.42-0.96)$ \\
\hline & \multicolumn{6}{|c|}{ P-heterogeneity $=0.89$} \\
\hline \multicolumn{7}{|l|}{ Fresh fruits } \\
\hline$\leqq$ once per week & $89(32.7)$ & $95(17.5)$ & Reference & $259(46.0)$ & $126(27.7)$ & Reference \\
\hline 2-4 time per week & $82(30.2)$ & $136(25.0)$ & $0.77(0.49-1.20)$ & $154(27.4)$ & $127(28.0)$ & $0.74(0.52-1.05)$ \\
\hline \multirow[t]{3}{*}{ Daily } & $101(37.1)$ & $312(57.5)$ & $0.50(0.33-0.76)$ & $150(26.6)$ & $201(44.3)$ & $0.60(0.42-0.85)$ \\
\hline & & & P-trend $=0.0009$ & & & P-trend $=0.004$ \\
\hline & \multicolumn{6}{|c|}{ P-heterogeneity $=0.92$} \\
\hline Non-daily & $171(62.9)$ & $231(42.5)$ & Reference & $413(73.4)$ & $253(55.7)$ & Reference \\
\hline \multirow[t]{2}{*}{ Daily } & $101(37.1)$ & $312(57.5)$ & $0.58(0.41-0.81)$ & $150(26.6)$ & $201(44.3)$ & $0.69(0.51-0.94)$ \\
\hline & \multicolumn{6}{|c|}{ P-heterogeneity $=0.92$} \\
\hline
\end{tabular}

${ }^{a} \mathrm{OR}$ and $95 \% \mathrm{CI}$ were calculated using unconditional logistic regression, adjusted for sex, age, education, cigarette smoking (pack-year categories), betel quid chewing (pack-year categories), and alcohol drinking (frequency).

studies from Iran, and 1 study from Indonesia) of upper aerodigestive tract (UADT) cancer (HNC + esophageal cancer) and found that the most consistent finding was a reduced UADT cancer risk associated with a diet rich in fruits and vegetables or nutrients contained in fruits and vegetables [5]. A large prospective cohort study with 490,802 participants from the United States by Freedman et al. reported an inverse association between total vegetable and fruit intake and $\mathrm{HNC}$ (hazard ratio for per serving $/$ day $/ 1000$ calories $=0.94,95 \%$ CI: 0.89 0.99 ) [9]. More recently, a case-control study conducted in a Chinese population reported that more frequent consumption of fruits and vegetables was associated with a reduced $\mathrm{HNC}$ risk [10]. Another recent case-control study from China reported a reduced oral cancer risk associated with frequent consumption of leafy vegetables, other vegetables, and fruits [11]. Our results together with evidence from published studies showed that vegetables and fruits are associated with a reduced HNC risk across all ethnicities.

Our results showed that the highest HNC risk occurred among individuals who had neither daily intake of vegetables nor daily intake of fruits. For those who had only daily intake of vegetables but no daily intake of fruits, their risk of HNC was lower than those who had neither daily intake of vegetables nor daily intake of fruits $(\mathrm{OR}=0.65,95 \%$ CI: 0.45-0.92), but was higher than those who had both daily intake of fruits and vegetables $(\mathrm{OR}=$ $1.45,95 \%$ CI: 1.14-1.84). This suggests that daily intake of fruits and daily intake of vegetables are both necessary to achieve the maximum benefit to reduce the risk of HNC.

Our analyses showed that the inverse association between consumption of fresh fruits and vegetables and HNC risk was not significantly different by the use of 
alcohol, betel quid, or cigarette. The pooled analysis by Chuang et al. also reported that the reduced risk of HNC associated with consumption of vegetables and fruits was not affected by the status of tobacco use or the intensity of alcohol consumption [6]. In a case-control study, Butler et al. showed that the intake of leafy vegetables was associated with a reduced HNC risk only among nonsmokers but not among smokers, whereas consumption of fruits was associated with a reduced $\mathrm{HNC}$ risk regardless of the smoking status [10]. In a large cohort study from the United States, Freedman et al. reported that the inverse association between total fruit and vegetable intake was similar by the status of smoking and alcohol use [9]. Overall, regardless of the use of alcohol, betel quid, or cigarette, consumption of fruits and vegetables appeared to remain inversely associated the HNC risk.

In our analysis, we found a significant association between consumption of egg and pharyngeal cancer. Due to the smaller number in the analysis stratified by HNC subsite, we could not rule out chance for this result. The inverse association between $\mathrm{HNC}$ and egg has been reported by other studies. Butler et al. reported a reduced HNC risk associated with egg consumption, particularly among nonsmokers [10]. Chen et al. reported that eating eggs less than 5 times per week was associated with an increased oral cancer risk (OR $=1.44,95 \% \mathrm{CI}$ : 1.21-1.71) [11]. In contrast, Chuang et al. reported that egg intake was associated with an increased $\mathrm{HNC}$ risk (highest quartile vs. lowest quartile: $\mathrm{OR}=1.48,95 \%$ CI: 1.20-1.82) [6]. Given the inconsistent results on the association between egg intake and HNC risk across studies, additional investigations are needed to clarify this association.

Processed meat, which is classified by the International Agency for Research on Cancer as "carcinogenic to humans" [12], has been associated with an increased HNC risk [6, 10]. However, our result did not show a significant association between processed meat and $\mathrm{HNC}$. The major reason for the null association in our study is likely due to the infrequent consumption of processed meat in our study population. Approximately $87 \%$ of our study participants reported eating processed meat once or less per week and only $2 \%$ reported daily consumption of processed meat.

This study has several limitations. First, the study participants were asked to recall their dietary habits in the year prior to the diagnosis of $\mathrm{HNC}$ or before the interview date (for controls). This might have introduced reverse-causality if the development of $\mathrm{HNC}$ had forced HNC patients to change their dietary habits. However, this short time-frame for recalling dietary habits had the advantage of reducing recall errors. Second, recall bias could be an issue in a case-control study because case subjects are often likely to ruminate more heavily on their past exposures that might cause their disease. As a result, recall bias could have biased our results away from the null and overestimated the association between diet (particularly consumption of vegetables and fruits) and HNC. However, because of public health campaigns, Taiwanese are more aware of the association between $\mathrm{HNC}$ and use of alcohol, betel quid, and cigarette, and less aware of the role of diet in the development of HNC. Therefore, recall bias in the association between diet and $\mathrm{HNC}$ is less likely in our study. Another limitation is that we asked about diet in broad categories instead of using a food frequency questionnaire with individual food items. The diet questions that we asked are a component of a comprehensive questionnaire that also surveys other exposure factors. To keep the interview at a reasonable length to minimize disruption to patients' hospital visits, we could only ask a limited number of questions on diet. Finally, information bias is another limitation because the dietary questionnaire we used was not validated. Consequently, the risk estimates generated by our study might be imprecise and might only reflect a general trend for the association between diet and HNC risk.

The major strength of the current study is that it provides important data for the role of diet in the risk of $\mathrm{HNC}$ in an East Asian population. The majority of the studies published to date on the association between diet and $\mathrm{HNC}$ were conducted in Western populations. Given the difference in dietary patterns, it is important to investigate the relationship between diet and HNC in different ethnic populations. Our results provide additional evidence to suggest that consumption of vegetables and fruits is beneficial in reducing HNC risk not only for Western populations but also for Taiwanese. Another strength of the current study is that we collected detailed data on the use of alcohol, betel quid, and cigarette, which are the three major risk factors of HNC. With this information, we were able to show that even after adjusting for alcohol, betel quid, and cigarette, consumption of fresh vegetables and fruits was still significantly associated with a reduced $\mathrm{HNC}$ risk. In addition, we were able to show that the inverse association between intake of fresh vegetables and fruits and HNC did not differ according to the status of alcohol, betel quid, or cigarette use. This suggests that consumption of vegetables and fruits might be beneficial in reducing $\mathrm{HNC}$ risk even for users of alcohol, betel quid, and cigarette.

In conclusion, the results of the current study supported an inverse association between consumption of fresh vegetables and fruits and $\mathrm{HNC}$ risk. In addition to cessation of cigarette smoking and betel quid chewing and reduction of alcohol drinking, a public health campaign for preventing the occurrence of $\mathrm{HNC}$ should also promote a healthy diet that contains plenty of fresh vegetables and fruits.

\section{MATERIALS AND METHODS}

The current study received an approval from the institutional review boards of the National Health Research Institutes and the National Cheng Kung University Hospital. Signed informed consent was obtained from each study participant. 


\section{Recruitment of study subjects}

The current analysis included data from an ongoing $\mathrm{HNC}$ case-control study that started subject recruitment on September 1, 2010 in the Department of Otolaryngology and the Department of Stomatology at the National Cheng Kung University Hospital. Eligible cases included individuals who were diagnosed with pathologically confirmed squamous cell carcinoma of the head and neck, including cancers of the oral cavity, oropharynx, hypopharynx, and larynx (ICD-10 codes: C00-C10, C12-C14, C32), had no history of any cancer diagnosis, were aged 20 years or older, and were able to provide informed consent. To compare the experience of risk factor exposure, controls frequency-matched to cases on sex- and age ( \pm 5 years) were recruited. Eligible controls were those who received surgery for non-cancerous conditions that are not associated with the use of alcohol, betel quid and cigarette, had no history of cancer diagnosis, and were aged 20 years or older.

\section{Data collection by interview}

A trained interviewer interviewed each study participant using a standardized questionnaire to collect information on dietary habits in the past year before the diagnosis of $\mathrm{HNC}$ for the case subjects or before the interview date for the control subjects. Each subject was asked about the frequency of consumption (ฏ once/ week, 2-4 times/week, or daily) for four plant-based food categories (fresh vegetables, pickled vegetables, fresh fruits, and citrus fruits) and five animal-based food categories (meat, processed meat, fish, egg, and dairy products). Data on potential confounders, including consumption of alcohol, betel quid, and cigarette, were also collected.

\section{Statistical analysis}

To compare the distributions of demographic characteristics (age, sex, and education) and lifestyle factors (use of alcohol, betel quid, and cigarette) between cases and controls, T-tests (for continuous variables) and chi-squared tests (for categorical variables) were performed.

The association between each food category and $\mathrm{HNC}$ risk was evaluated by an OR and $95 \% \mathrm{CI}$ calculated using unconditional logistic regression, adjusted for sex, age, educational level, alcohol drinking (frequency), betel quid chewing (pack-years), and cigarette smoking (packyears). The pack-year of cigarette smoking $=$ (number of cigarettes smoked per day/20) x total number of year of cigarette smoking. The pack-year of betel quid chewing $=$ (number of betel quids chewed per day/20) x total number of year of betel quid chewing.

Additional analyses were performed with consumption of fresh vegetables and fruits, which were the food categories that showed a significant inverse association with $\mathrm{HNC}$ risk in our analysis. We examined the joint effect of fresh vegetables and fruits by examining the risk of $\mathrm{HNC}$ associated with different combinations of fresh vegetable and fruit consumption (yes to daily intake of vegetables/yes to daily intake of fruits; yes to daily intake of vegetables/no to daily intake of fruits; no to daily intake of vegetables/yes to daily intake of fruits; no to daily intake of vegetables/no to daily intake of fruits). Further analyses were conducted to examine the association between consumption of fresh fruits and vegetables stratified by the use of alcohol, betel quid, or cigarette to determine whether the relationship between consumption of fresh fruits and vegetables and HNC risk might differ according to the use of alcohol, betel quid, and cigarette. Heterogeneity between strata of alcohol, betel quid, or cigarette use was evaluated with loglikelihood ratio test comparing the unconditional logistic regression model with the product term (vegetables or fruits $\mathrm{x}$ alcohol, vegetables or fruits $\mathrm{x}$ betel quid, or vegetables or fruits $x$ cigarette) to the model without the product term.

\section{CONFLICTS OF INTEREST}

The authors declare no conflicts of interest.

\section{FUNDING}

This work was supported by the Establishment of Cancer Research System Excellence Program funded by the Ministry of Health and Welfare, Taiwan (MOHW106TDU-B-211-144-004, MOHW105-TDU-B-212-134-013) and by the National Health Research Institutes (CA-106SP-01).

\section{REFERENCES}

1. Ferlay J, Soerjomataram I, Ervik M, Dikshit R, Eser S, Mathers C, Rebelo M, Parkin DM, Forman D, Bray F. GLOBOCAN 2012 v1.0, Cancer Incidence and Mortality Worldwide: IARC CancerBase No. 11. Lyon, France: IARC Press; 2013. Accessed on October 15, 2015. http://globocan. iarc.fr.

2. Health Promotion Administration. Ministry of Health and Welfare, Taiwan. Cancer Registry Annual Report, 2014. https://www.hpa.gov.tw/Pages/Detail. aspx?nodeid=269\&pid=7330 accessed on June 22, 2017.

3. Boyle P, Levin B. World Cancer Report. Lyon, France: IARC Press; 2008.

4. Young D, Xiao CC, Murphy B, Moore M, Fakhry C, Day TA. Increase in head and neck cancer in younger patients due to human papillomavirus (HPV). Oral Oncol. 2015; 51:727-30. 
5. Bravi F, Edefonti V, Randi G, Ferraroni M, La Vecchia C, Decarli A. Dietary patterns and upper aerodigestive tract cancers: an overview and review. Ann Oncol. 2012; 23:3024-39.

6. Chuang SC, Jenab M, Heck JE, Bosetti C, Talamini R, Matsuo K, Castellsague X, Franceschi S, Herrero R, Winn DM, La Vecchia C, Morgenstern H, Zhang ZF, et al. Diet and the risk of head and neck cancer: a pooled analysis in the INHANCE consortium. Cancer Causes Control. 2012; 23:69-88.

7. Pan WH, Wu HJ, Yeh CJ, Chuang SY, Chang HY, Yeh NH, Hsieh YT. Diet and health trends in Taiwan: comparison of two nutrition and health surveys from 1993-1996 and 20052008. Asia Pac J Clin Nutr. 2011; 20:238-50.

8. Wu YH, Yen CJ, Hsiao JR, Ou CY, Huang JS, Wong TY, Tsai ST, Huang CC, Lee WT, Chen KC, Fang SY, Wu JL, Hsueh WT, et al. A Comprehensive Analysis on the Association between Tobacco-Free Betel Quid and Risk of Head and Neck Cancer in Taiwanese Men. PLoS One. 2016; 11:e0164937.
9. Freedman ND, Park Y, Subar AF, Hollenbeck AR, Leitzmann MF, Schatzkin A, Abnet CC. Fruit and vegetable intake and head and neck cancer risk in a large United States prospective cohort study. Int J Cancer. 2008; 122:2330-6.

10. Butler C, Lee YA, Li S, Li Q, Chen CJ, Hsu WL, Lou PJ, Zhu C, Pan J, Shen H, Ma H, Cai L, He B, et al. Diet and the risk of head-and-neck cancer among never-smokers and smokers in a Chinese population. Cancer Epidemiol. 2017; 46:20-6.

11. Chen F, Yan L, Lin L, Liu F, Qiu Y, Wang J, Wu J, Liu F, Huang J, Cai L, He B. Dietary score and the risk of oral cancer: a case-control study in southeast China. Oncotarget. 2017; 8:34610-6. https://doi.org/10.18632/ oncotarget.16659.

12. Bouvard V, Loomis D, Guyton KZ, Grosse Y, Ghissassi FE, Benbrahim-Tallaa L, Guha N, Mattock H, Straif K. Carcinogenicity of consumption of red and processed meat. Lancet Oncol. 2015; 16:1599-600. 